\title{
Nieren und Eisenspiegel im Blick behalten
}

Fragestellung: Mit welchen anderen Erkrankungen ist das Restless-legs-Syndrom assoziiert?

Hintergrund: Das Restless-legs-Syndrom (RLS) ist ein sehr interessantes Krankheitsbild, das zum ersten Mal vor 25 Jahren beschrieben und diagnostiziert wurde. Seitdem wurde eine ganze Reihe von Klassifikationen eingeführt, die zuletzt im Jahr 2014 aktualisiert wurden. In der Literatur wurde eine Vielzahl von Assoziationen des RLS mit anderen neurologischen und nicht neurologischen Erkrankungen beschrieben, was die diagnostische Zuordnung des primär zu einem unterstellten sekundären RLS erschwert. Die Arbeitsgruppe um Professor Claudia Trenkwalder aus Kassel hat in Zusammenarbeit mit den neurologischen Kliniken in Innsbruck und München eine systematische Literaturrecherche zu diesem Thema durchgeführt.

Patienten und Methodik: Die Autoren identifizierten Studien zum Zusammenhang zwischen RLS und Eisenmangel, Anämie, Erkrankungen der Nieren, arterieller Hypertonie und kardiovaskulären Erkrankungen. Bei den neurologischen Erkrankungen wurden Assoziationen mit Kopfschmerz, Schlaganfall, neurodegenerativen Erkrankungen, Polyneuropathie und Multiple Sklerose untersucht. Anschließend wurde die Assoziation mit dem RLS mit hoher, mittelstarker und geringer Wahrscheinlichkeit eingestuft.

Ergebnisse: Die Auswertung der gesichteten Literatur ergab nur eine gesicherte Assoziation des RLS mit Eisenmangel und Nierenerkrankungen. Bei den anderen Erkrankungen war die Assoziation gering und konnte letztendlich nicht ausgeschlossen werden. Unwahrscheinlich ist eine Assoziation mit einer Anämie ohne Eisenmangel, mit Lungenerkrankungen, Multipler Sklerose, Kopfschmerzen, Schlaganfall,

Trenkwalder C, Allen R, Högl B et al. Restless legs syndrome associated with major diseases: A systematic review and new concept. Neurology 2016; 86: $1336-43$
Narkolepsie und Ataxien.

Schlussfolgerungen: Eine systematische Literaturrecherche ergab ausschließlich eine hohe Assoziation von Eisenmangel und Nierenerkran kungen mit dem RLS. Dies hat auch therapeutische Konsequenzen.

\section{- Kommentar von Hans-Christoph Diener, Essen}

\section{Bei RLS-Patienten Eisenmangel ausgleichen}

Die systematische Literaturrecherche ist sehr hilfreich, da basierend auf diesen Ergebnissen ein neues Modell für das RLS vorgeschlagen wird. Dieses Modell umfasst genetische Formen des RLS ohne Komorbidität, einen Überlappungsbereich, in dem das RLS durch andere Erkrankungen verstärkt wird, und wahrscheinlich eine geringe Anzahl von Erkrankungen, bei denen nur die Begleiterkrankung das RLS erklärt.

Die praxisrelevanten Erkenntnisse aus diesen Studien sind, dass ein Eisenmangel beim RLS ausgeglichen und dass auch eine Nierenerkrankung soweit wie möglich behandelt werden sollte. 